\title{
Painéis MDP produzidos com resíduos de extração de celulose
}

\author{
Nayhara Camila Andrade ${ }^{1} \oplus$, Ticyane Pereira Freire Sabino ${ }^{1} \oplus$, Isabelle Cristine de Carvalho Terra ${ }^{1} \oplus$, \\ Lourival Marin Mendes ${ }^{1} \odot$, Rafael Farinassi Mendes ${ }^{1} \oplus$

\footnotetext{
1 Universidade Federal de Lavras, Lavras, MG, Brasil. E-mail: nayhara_andrade10@hotmail.com; ticyane-pf@hotmail.com; belsterra@gmail.com; lourival@dcf.ufla.br; rafael.mendes@deg.ufla.br
}

RESUMO: Muitas alternativas vêm sendo estudadas sobre o emprego de resíduos agrícolas, agroindustriais e florestais na produção de painéis aglomerados na tentativa de aproveitar os resíduos oriundos desses ramos, que por muitas vezes geram problemas ambientais devido à sua destinação incorreta. Dessa forma, este trabalho teve por objetivo avaliar o potencial de utilização do resíduo gerado durante o processo de produção da celulose (UKP), na fabricação de painéis MDP (Medium Density Particleboard). As proporções utilizadas para a substituição da madeira de Pinus oocarpa pelo resíduo foram de 15, 30, $45 \mathrm{e}$ $60 \%$ (em massa), utilizando o adesivo ureia-formaldeído. Foram realizados os ensaios de densidade aparente (DA), absorção de água após duas e vinte e quatro horas de imersão (AA2h e AA24h), inchamento em espessura após duas e vinte e quatro horas de imersão (IE2h e IE24h), ligação interna (LI), e módulo de elasticidade (MOE) e módulo de ruptura (MOR) à flexão estática. A utilização de partículas de resíduo UKP para a produção de painéis MDP se mostrou prejudicial, uma vez que acarretou na diminuição das propriedades mecânicas e no aumento dos valores de absorção de água e inchamento em espessura.

Palavras-chave: material lignocelulósico; propriedades físico-mecânicas; ureia-formaldeído

\section{MDP panels produced with pulp extraction residues}

ABSTRACT: Many alternatives have been studied on the use of agricultural, agroindustrial and forest residues in the production of particleboard in an attempt to take advantage of the residues from these branches, which often generate environmental problems due to their incorrect destination. Thus, the objective of this work was to evaluate the potential of the waste generated during the pulp production process (UKP) in the manufacture of MDP panels. The proportions used for the replacement of Pinus oocarpa wood by the residue were $0,15,30,45$ and $60 \%$ (by mass), using the urea-formaldehyde binder. The apparent density (DA), water absorption after two and twenty four hours of immersion (AA2h and AA24h), thickness swelling after two and twenty four hours of immersion (IE2h and IE24h), internal LI), and modulus of elasticity (MOE) and modulus of rupture (MOR) to static bending. The use of UKP residue particles for the production of MDP panels proved to be detrimental since it resulted in a decrease in mechanical properties and an increase in water absorption values and thickness swelling.

Key words: lignocellulosic material; physical-mechanical properties; urea-formaldehyde 


\section{Introdução}

Os painéis aglomerados são formados por componentes de desagregação da madeira, possuindo forma e tamanhos variados, que são reconstituídos por meio de adesivos e prensagem com temperatura (Iwakiri, 2005). Dentre esses produtos reconstituídos de madeira estão os painéis MDP (Medium Density Particleboard), esses painéis possuem três camadas, sendo duas de partículas menores nas faces e uma de partículas maiores no miolo do painel. Podem ser fabricados a partir de uma variedade de materiais e são largamente empregados na indústria moveleira (Mesquita et al., 2015).

Em 2018, o segmento brasileiro de painéis de madeira, ocupou o 80 lugar no ranking mundial de maiores produtores, possuindo mais de $3 \%$ do volume total da produção mundial. A produção nacional de painéis reconstituídos em 2018 foi de 7,9 milhões de metros cúbicos (Ibá, 2018). Torna-se evidente por meio desses dados, a importância do setor de painéis para a cadeia produtiva florestal e a imprescindibilidade de pesquisas científicas que contribuam com o crescimento e com o desenvolvimento de inovações tecnológicas relacionadas às indústrias de painéis reconstituídos.

Na produção de painéis de madeira reconstituída podem ser utilizados todo e qualquer material lignocelulósico, tornando-se possível a criação de um material com características sustentáveis e excelentes propriedades físico-mecânicas (Fiorelli et al., 2012). Dessa forma, vários pesquisadores têm apresentado trabalhos sobre o emprego de resíduos agrícolas, agroindustriais e florestais na produção de painéis aglomerados, como o bagaço de cana-de-açúcar (Mesquita et al., 2018; Nakanishi et al., 2018); sabugo de milho (Scatolino et al., 2013); casca de mamona (Silva et al., 2018); casca de arroz (César et al., 2017); bagaço de sorgo (Guimarães Junior et al., 2016); pergaminho do grão de café (Scatolino et al., 2017); podas de erva mate (Carvalho et al., 2015); pseudocaule da bananeira (Guimarães et al., 2014) e palha de milho (Silva et al., 2015).

Entre as diversas atividades florestais, o setor de papel e celulose brasileiro é destaque mundial, apresentando um crescimento de $115 \%$, no acumulado dos últimos 50 anos, partindo de 80 milhões de toneladas no ano de 1967 para pouco mais de 170 milhões de toneladas no ano de 2017 (Consufor, 2018). O Brasil, ocupa a segunda posição entre os maiores produtores mundiais de celulose, produzindo cerca de 19,5 milhões de toneladas (Ibá, 2018).

O processo de produção da celulose é responsável pela geração de vários resíduos industriais, tais como o lodo biológico oriundo do tratamento de efluentes, as cascas de Eucalipto, a lama de cal, além dos rejeitos da depuração da polpa marrom e branqueada (Unbleach kraft pulp/Bleach kraft pulp - UKP/BKP) (Gama et al., 2010). Para produção de uma tonelada de polpa celulósica não branqueada são gerados cerca de $16 \mathrm{~kg}$ de resíduos UKP/BKP da polpação, fato este que atesta a necessidade de se encontrar uma destinação adequada para eles (Cenibra, 2008).
Os resíduos UKP/BKP possuem uma grande quantidade de fibras de excelente qualidade, que apresentam um grande potencial de comercialização. No entanto, atualmente, esses resíduos são descartados de formas inadequadas em aterros sanitários ou levados para compostagem, contribuindo para sérios problemas ambientais. A utilização desse resíduo na produção de painéis aglomerados, é uma excelente alternativa, uma vez que agrega valor à um produto que seria descartado, e contribui com a preservação do meio ambiente.

A incorporação do resíduo fibroso da polpação (UKP) em painéis MDP, possibilita uma nova forma de seu aproveitamento, agregando valor a um material de descarte, proporcionando uma destinação adequada e a geração de um novo produto no mercado. Dessa forma, este trabalho teve por objetivo avaliar o efeito da utilização de resíduos UKP em associação a madeira de Pinus oocarpa, com o emprego de adesivo ureia-formaldeído, sobre as propriedades de painéis MDP, permitindo uma destinação adequada do resíduo e agregando valor a ele.

\section{Material e Métodos}

Matéria-prima utilizada e caracterização química e física do resíduo e da madeira

Para a produção dos painéis foram utilizadas partículas de madeira de Pinus oocarpa com idade de 28 anos, extraída no campus da Universidade Federal de Lavras. Já o resíduo fibroso da polpação (UKP) foi fornecido pela empresa Cenibra da cidade de Belo Oriente - MG, proveniente de um híbrido de Eucalyptus uroplylla e Eucalyptus grandis, com 7 anos de idade.

O resíduo e a madeira foram preparados em moinho do tipo Willey e separados em peneiras sobrepostas de 40 e 60 mesh, sendo utilizado para análise apenas o material retido na peneira de 60 mesh. As amostras foram então armazenadas em sacos abertos e levadas à sala de climatização com condições de temperatura de $20 \pm 2{ }^{\circ} \mathrm{C}$ e umidade de $65 \pm 3 \%$, até o momento em que apresentaram massa constante. A análise química foi realizada de acordo com as metodologias apresentadas na Tabela 1.

Para determinação da densidade básica dos materiais lignocelulósicos se utilizou a norma NBR 11941 (ABNT, 2003), com adaptações.

Para a determinação da densidade básica da madeira, foram retirados discos de $5 \mathrm{~cm}$ de espessura na base, a 25, 50, 75 e $100 \%$ da altura comercial das árvores (diâmetro mínimo de $5 \mathrm{~cm}$ ). Todo procedimento foi realizado de acordo com a NBR 11941 (ABNT, 2003).

Tabela 1. Metodologias para a análise química do resíduo e madeira utilizada no experimento.

\begin{tabular}{cc}
\hline Componente & Metodologia utilizada \\
\hline Teores de Lignina & ABNT 7989 (ABNT, 2010) \\
Extrativos totais & ABNT 14853 (ABNT, 2010) \\
Cinzas & ABNT 13999 (ABNT, 2003) \\
Holocelulose & Obtida por diferença \\
\hline
\end{tabular}


Processamento do material, produção e avaliação dos painéis

As árvores de Pinus oocarpa coletadas no campus da Universidade Federal de Lavras (UFLA) foram cortadas com dimensões de 1,2 $\mathrm{m}$ e imersas em tanque com água por um período de 10 dias antes do processamento, com o objetivo de evitar o ataque de organismos xilófagos e fungos, além de prevenir o surgimento de rachaduras de topo.

Para a obtenção das lâminas de madeira, as toras foram levadas a um tanque de aquecimento com água a temperatura de $60{ }^{\circ} \mathrm{C}$ por um período de $24 \mathrm{~h}$. Posteriormente, as toras foram divididas no centro e então laminadas em um torno laminador e secas à temperatura ambiente por um período de $24 \mathrm{~h}$.

Após a secagem, as lâminas passaram por um moinho de facas e foram peneiradas. Nas faces do painel foram utilizadas as partículas que passaram pela peneira com abertura de 40 mesh e ficaram retidas na peneira de 60 mesh. Para o miolo foram empregadas as partículas que passaram pela peneira de 12 mesh e ficaram retidas na de 20 mesh.

A análise das propriedades do adesivo ureia-formaldeído utilizado para produção dos painéis MDP foi realizada de acordo com as metodologias apresentadas na Tabela 2.

Na produção dos painéis MDP, as partículas do resíduo UKP foram utilizadas como substituição nas proporções de 15, $30 \%, 45 \%$ e $60 \%$ em relação ao peso total de partículas do miolo. Para cada composição foram produzidos três painéis.

A aplicação do adesivo ureia-formaldeído nas partículas de madeira e resíduos UKP foi realizada por aspersão em uma encoladeira do tipo tambor giratório, sendo aplicado $11 \%$ para a face dos painéis e $7 \%$ para o miolo (base massa seca das partículas). Após 8 minutos de mistura na encoladeira, as partículas foram conduzidas para uma caixa formadora de colchão com dimensão de $30 \times 30 \mathrm{~cm}$.

A pré-prensagem do colchão foi realizada com a aplicação de 0,4 MPa em uma prensa manual, com objetivo de proporcionar uma melhor conformação no painel e facilitar o carregamento para a posterior prensagem a quente, a qual se realizou na temperatura de $165^{\circ} \mathrm{C}, 4 \mathrm{MPa}$ de pressão e tempo

Tabela 2. Metodologias utilizadas na avaliação do adesivo ureia-formaldeído utilizado para produção dos painéis MDP.

\begin{tabular}{cc}
\hline Propriedades & Metodologia utilizada \\
\hline Teor de Sólidos & ASTM D1582 (ASTM, 1994) \\
Viscosidade & ASTM D1200 (ASTM, 1994) \\
pH & pH-metro \\
Tempo de Gelatinização "Gel Time" & Mori (2000) \\
\hline
\end{tabular}

de prensagem de 8 minutos. Os painéis foram produzidos com espessura de $1,5 \mathrm{~cm}$.

Os painéis ficaram acondicionados em uma sala de climatização com uma temperatura de $20 \pm 2{ }^{\circ} \mathrm{C}$ e umidade de $65 \pm 3 \%$. O processo de retirada dos corpos-de-prova foi realizado com o uso de uma serra circular. Os painéis MDP foram ensaiados após atingirem massa constante. Na Tabela 3 é possível observar os ensaios e as normas adotadas neste trabalho para a avaliação das propriedades físicas e mecânicas dos painéis.

Os dados físicos e mecânicos foram avaliados se considerando um delineamento inteiramente casualizado. A composição química foi avaliada por análise de variância a 5\% de significância. As propriedades de densidade e razão de compactação foram avaliadas por análise de variância e teste de média Scott-Knott, ambos a 5\% de significância. As propriedades de umidade, absorção de água, inchamento em espessura, ligação interna e módulos de ruptura e de elasticidade à flexão estática foram avaliados por análise de variância e de regressão, ambos a 5 e 1\% de significância.

\section{Resultados e Discussão}

\section{Propriedades das partículas de resíduo UKP e da madeira de Pinus oocarpa}

$\mathrm{Na}$ Tabela 4, é possível observar que os valores de extrativos foram superiores para o resíduo UKP comparado à madeira. Em termos de adesão, o teor de extrativos é o valor mais relevante, pois altos teores desses componentes podem bloquear o contato do adesivo com as partículas no processo de colagem (Bufalino et al., 2012). Mendes et al. (2014), encontrou para Pinus oocarpa um valor médio de $6,83 \%$ de extrativos totais, sendo um pouco superior ao valor observado neste trabalho, o que pode ter ocorrido devido a diferença de local de plantio do material. Esses baixos valores de extrativos encontrados para Pinus oocarpa, a principal matéria prima utilizada pela indústria de painéis aglomerados, comprovam a sua viabilidade de utilização na produção de painéis aglomerados.

De acordo com Bufalino et al. (2012), a lignina é um adesivo natural e, portanto, maiores teores são desejáveis para a produção de painéis de madeira reconstituída, uma vez que contribui para os mecanismos de adesão, agindo como amortecedor das microfibrilas de celulose, limitando o movimento paralelo à grã, colaborando com o processo de colagem das partículas. Uma baixa quantidade de

Tabela 3. Metodologia para as propriedades físicas e mecânicas dos painéis MDP.

\begin{tabular}{lc}
\hline \multicolumn{1}{c}{ Teste } & Painéis MDP \\
\hline \multicolumn{1}{c}{ Metodologia } \\
\hline Absorção de água após 2h de imersão (AA_2h-24h) & ASTM D-1037 (ASTM, 2016) \\
Inchamento em espessura após 2h de imersão (IE_2h-24h) & ASTM D-1037 (ASTM, 2016) \\
Umidade e Densidade aparente & NBR 14810-3 (ABNT, 2013) \\
Flexão estática - Módulo de elasticidade (MOE) e Módulo de ruptura (MOR) & DIN-52362 (DIN, 1982) \\
Ligação Interna (LI) & NBR 14810-2 (ABNT, 2018) \\
Razão de compactação & Densidade do painel/densidadedo material \\
\hline
\end{tabular}


Tabela 4. Composição química do resíduo UKP e da madeira de Pinus oocarpa.

\begin{tabular}{ccccc}
\hline & Extrativos & Lignina & Cinzas & Holocelulose \\
\cline { 2 - 5 } & & & $(\%)$ & \\
\hline Pinus oocarpa & $4,72^{(0,04)} \mathrm{A}$ & $31,32^{(1,17)} \mathrm{A}$ & $0,20^{(0,03)} \mathrm{A}$ & $63,71^{(1,12)} \mathrm{A}$ \\
Resíduo UKP & $15,75^{(0,63)} \mathrm{B}$ & $16,10^{(1,79)} \mathrm{B}$ & $3,87^{(1,05)} \mathrm{B}$ & $64,28^{(1,33)} \mathrm{A}$ \\
\hline
\end{tabular}

Médias seguidas de mesma letra, na coluna, não diferem entre si pelo teste $\mathrm{F}$, a um nível de significância de $5 \%$. Os valores entre parênteses correspondem ao desvio padrão.

lignina foi encontrada para o resíduo UKP $(15,75 \%)$, devido, provavelmente, à sua remoção pelo processo de cozimento da indústria de celulose.

Não foi observada diferenciação estatística para a quantidade de holocelulose da madeira e do resíduo UKP. A holocelulose, fração de carboidrato da madeira, apresenta grupos hidroxílicos livres, principalmente na região amorfa da celulose e hemiceluloses, que são as principais responsáveis pela ligação com as moléculas de água, afetando a absorção de água dos painéis (Guimarães et al., 2013).

Em relação à quantidade de cinzas, a madeira apresentou o menor valor comparado ao UKP. Soares et al. (2017), afirma que altas porcentagens de cinzas provocam alterações indesejáveis nos painéis. $O$ teor de cinzas, os quais estão como componente inorgânicos no material lignocelulósicos, pode afetar $\mathrm{o} \mathrm{pH}$ do material, causando a pré-cura da resina, desse modo, o alto teor de componentes inorgânicos encontrado para o resíduo UKP, pode interferir negativamente, prejudicando o grau de adesão entre as partículas, reduzindo as propriedades mecânicas dos painéis.

\section{Propriedades físicas dos painéis MDP}

A densidade básica da madeira e do resíduo UKP foram de $456 \pm 14 \mathrm{~kg} \mathrm{~m}^{-3}$ e $325 \pm 90 \mathrm{~kg} \mathrm{~m}^{-3}$, respectivamente. Não houve diferença significativa entre os tratamentos avaliados, a densidade aparente dos painéis MDP variou de 594 a 615 $\mathrm{kg} \mathrm{m}^{-3}$ (Tabela 5). De acordo com a norma NBR 14810 (ABNT, 2006), que estabelece valores de classificação em função da densidade dos painéis, todos os painéis produzidos são classificados como painéis de média densidade (maior que $590 \mathrm{~kg} \mathrm{~m}^{-3}$ ).

Houve diferença significativa para razão de compactação entre os tratamentos analisados, todos os painéis com adição do resíduo se diferenciaram do controle (Tabela 5). Nas porcentagens de 15 e $30 \%$ de resíduo não foi observada diferença estatística entre os tratamentos, contudo se diferenciaram dos tratamentos onde se aplicou 45 e $60 \%$

Tabela 5. Valores médios de densidade aparente dos painéis MDP.

\begin{tabular}{ccc}
\hline $\begin{array}{c}\text { Porcentagem } \\
\text { de resíduo UKP } \\
(\%)\end{array}$ & $\begin{array}{c}\text { Densidade } \\
\text { aparente } \\
\left(\mathbf{k g ~ m}^{-3}\right)\end{array}$ & $\begin{array}{c}\text { Razão } \\
\text { de compactação }\end{array}$ \\
\hline 0 & $0,594^{(0,09)} \mathrm{A}$ & $1,303^{(0,08)} \mathrm{C}$ \\
15 & $0,615^{(0,05)} \mathrm{A}$ & $1,409^{(0,06)} \mathrm{B}$ \\
30 & $0,602^{(0,08)} \mathrm{A}$ & $1,445^{(0,04)} \mathrm{B}$ \\
45 & $0,614^{(0,04)} \mathrm{A}$ & $1,546^{(0,04)} \mathrm{A}$ \\
60 & $0,601^{(0,04)} \mathrm{A}$ & $1,592^{(0,05)} \mathrm{A}$ \\
\hline
\end{tabular}

Médias seguidas de mesma letra, na coluna, não diferem entre si pelo teste Scott-Knott, a um nível de significância de $5 \%$. Os valores entre parênteses correspondem ao desvio padrão. de resíduo. A aplicação de 45 e $60 \%$ de resíduo também se mostraram iguais estatisticamente, obtendo os maiores valores médios de razão de compactação.

A relação entre a densidade do painel e a densidade do material lignocelulósico, definida como razão de compactação, deve estar dentro da faixa ideal de 1,3 e 1,6 para garantir uma área de contato satisfatória entre as partículas e densificação suficiente para a formação do painel (Moslemi, 1974; Maloney, 1993). Todos os valores de razão de compactação obtidos pelos tratamentos se apresentaram dentro da faixa ideal, sendo observado o aumento dos valores médios a medida que aumentou a porcentagem de utilização de resíduo UKP. Quando se utiliza resíduo agrícola e/ou florestal na produção de painéis, essa razão de compactação tende a ser maior uma vez que a densidade desses resíduos é menor que as partículas de madeira (Mesquita et al., 2015; Guimarães Junior et al., 2016; Scatolino et al., 2017; Silva et al., 2018).

Na Figura 1, observa-se que não houve efeito significativo da porcentagem de utilização de resíduo de UKP sobre a umidade dos painéis. A norma brasileira NBR 14810 (ABNT, 2013) estipula valores de umidade entre 5 e 11\%, portanto os cinco tratamentos atenderam as especificações da norma.

Na Figura 2, observa-se que houve efeito significativo da porcentagem de resíduos UKP sobre as propriedades de absorção de água após 2 e 24 h, com aumento linear nos valores médios de $\mathrm{AA} 2 \mathrm{~h}$ e $\mathrm{AA} 24 \mathrm{~h}$ à medida em que se acrescentou uma maior porcentagem de resíduos UKP.

Esse aumento nos valores médios de absorção de água em função de maior porcentagem de resíduos UKP, pode estar relacionado ao caráter hidrofílico do resíduo de extração de

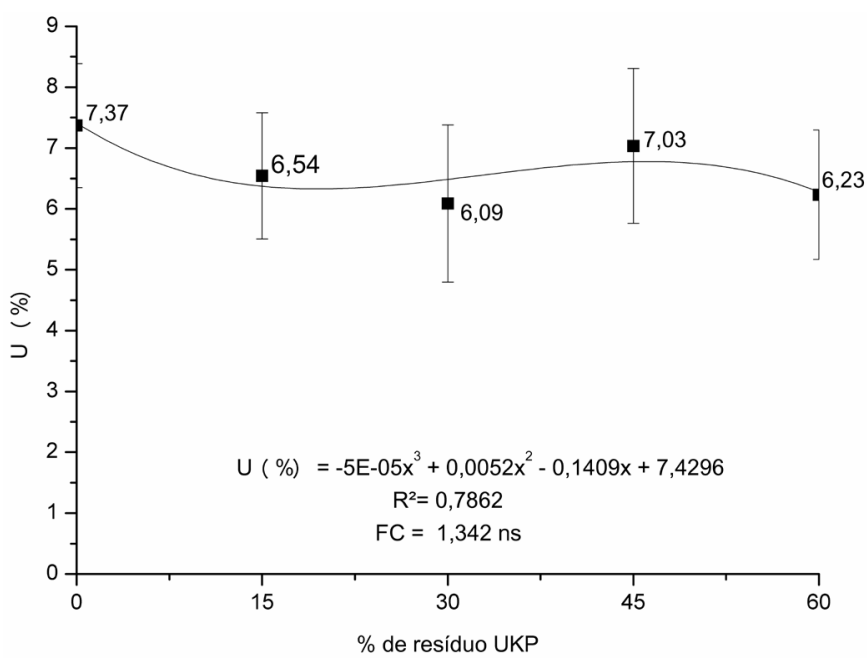

ns - Análise de regressão não significativa ao nível de $1 \%$.

Figura 1. Valores médios de umidade dos painéis MDP com resíduo UKP. 


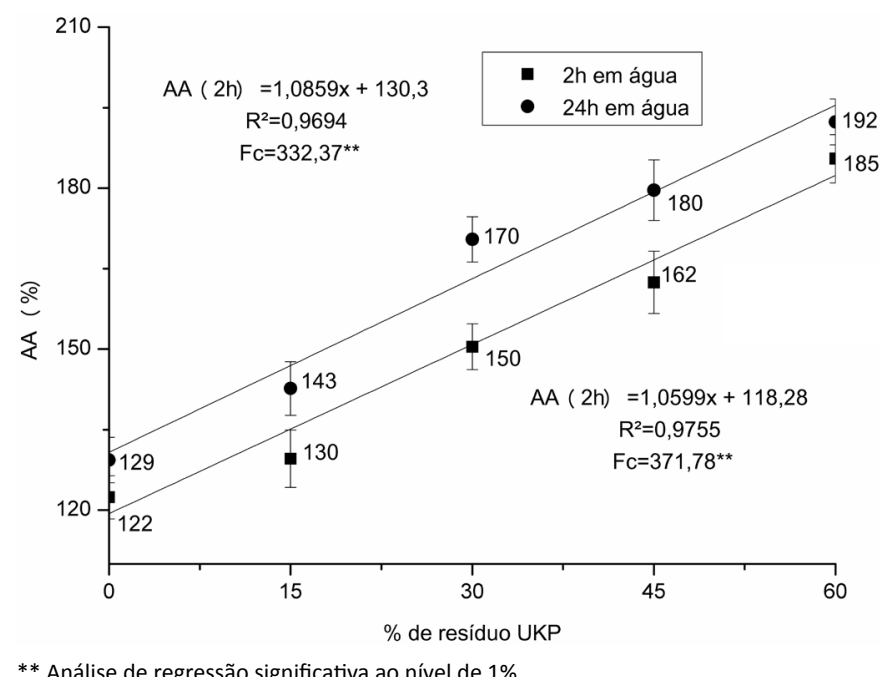

Figura 2. Valores médios de absorção de água em $2 \mathrm{~h}$ e $24 \mathrm{~h}$ dos painéis MDP com resíduo UKP.

celulose utilizado nas camadas mais internas dos painéis MDP, devido a menor porcentagem de lignina (Tabela 3) presente no resíduo UKP comparado à madeira de pinus, apresentando menor resistência à água. Além disso, esse aumento pode estar ligado ao não uso de aditivos, a fim de diminuir a afinidade do painel com a água, como é o caso da emulsão de parafina. $\mathrm{O}$ alto teor de extrativos encontrado para o resíduo UKP, também afetou essa característica. Nemli et al. (2018) afirmam que materiais com elevados teores de extrativos apresentam dificuldades de colagem, resultando em baixa resistência da ligação adesiva entre as partículas, acarretando maior número de poros entre elas.

Com o aumento da porcentagem de resíduo, houve um aumento na razão de compactação, o que contribuiu com o acréscimo nos valores de absorção de água dos painéis.

Esse aumento de absorção de água pelos painéis com porcentagem maiores de resíduos pode ser explicada pela densidade reduzida do material (UKP), onde para se obter igual densidade nominal do painel, se faz necessário um maior número de partículas em comparação com materiais de maior densidade, acarretando na presença de um maior número de sítios higroscópicos e, gerando portanto, maior absorção de água.

Na produção de painéis com diferentes porcentagens de madeira de Eucalyptus grandis (0, 20, 40,60, 80 e 100\%) e casca de arroz (0, 20, 40, 60, 80 e 100\%), Melo et al. (2009) observaram comportamento equivalente ao apresentado na presente pesquisa, em que à medida que se aumentou a porcentagem de resíduos, ocasionou em aumento na absorção de água após 2 e $24 \mathrm{~h}$ de imersão em água. Morais et al. (2018), na produção de painéis com diferentes porcentagens $(0,25$, 50, 75 e 100\%) de Bambusa tuldoides e Eucalyptus grandis, também observaram um aumento na absorção de água para 2 e $24 \mathrm{~h}$ de imersão.

Houve efeito significativo das porcentagens de resíduos UKP sobre o inchamento em espessura após 2 e $24 \mathrm{~h}$ de imersão em água (Figura 3). Foi observado aumento linear dos

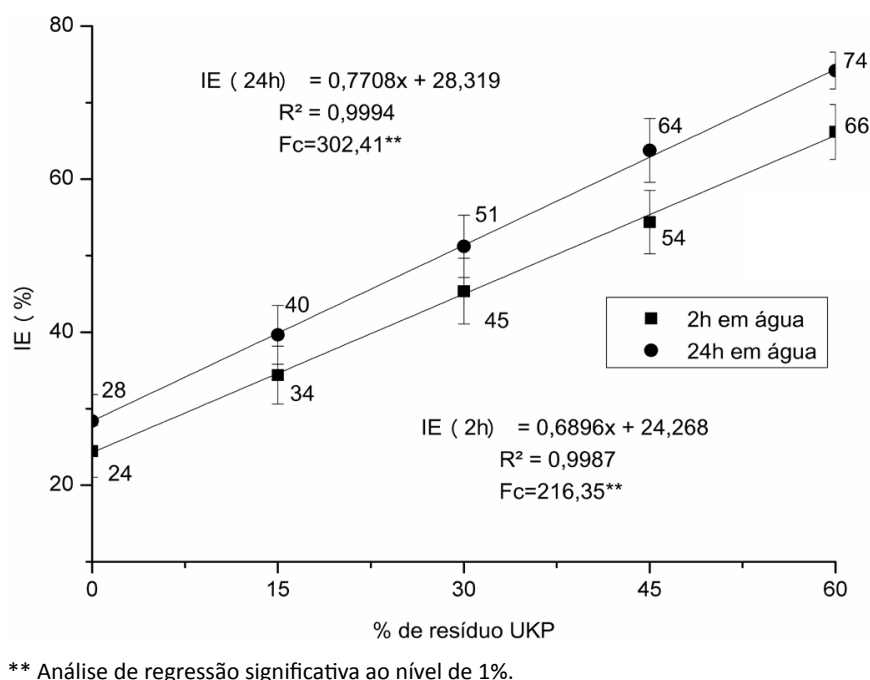

Figura 3. Valores médios de inchamento em espessura $2 \mathrm{~h}$ e 24h dos painéis MDP com resíduo UKP.

valores de inchamento dos painéis em função da porcentagem de resíduo de UKP utilizado. Esse resultado indica que quanto maior a porcentagem de resíduo empregada, maior será a instabilidade dimensional, proporcionando também maior absorção de água dos painéis, em função do aumento do número de poros.

O aumento da razão de compactação com a utilização de materiais de menor densidade acarreta em maior quantidade de partículas compactadas, que quando em contato com a água, liberam tensões da prensagem, originando um maior número de sítios hidroxílicos que se ligam com a água, promovendo maior inchamento em espessura (Guimarães Junior et al., 2016; Scatolino et al., 2017; Sulaiman, et al., 2018). Esse fato ainda está relacionado com a menor quantidade de lignina e pela maior quantidade de extrativos (Tabela 3 ), o que prejudica a ligação entre as partículas (Figura 4) e permite maior liberação das tensões de prensagem e descolamento entre as partículas, proporcionando aumento dos valores de inchamento em espessura (Xiaodi \& Minghui, 2018).

Essa tendência crescente nos valores de inchamento em função do aumento do resíduo nos painéis também foi relatada por outros autores. Mendes et al. (2010) estudaram o emprego de casca de café $(25,50$ e $75 \%)$ em painéis de Eucalyptus urophylla e também observaram aumento nos valores de IE24h com o da porcentagem de casca. Mendes et al. (2018), em estudo com painéis aglomerados produzidos com diferentes posições radiais de madeira de Pinus oocarpa, observaram que a razão de compactação da região interna do painel foi estatisticamente diferente das demais regiões, apresentando o maior valor médio, como consequência da menor densidade da madeira presente na região interna do painel; resultado este semelhante ao obtido no presente estudo, uma vez que resíduo com baixa densidade foi utilizado, acarretando em um aumento na razão de compactação e, consequentemente, em um elevado valor para inchamento em espessura.

O valor máximo para IE24h em painéis do tipo 3 e tipo 4 é de $14 \%$ para painéis MDP de uso geral em condições úmidas, 
estabelecido pela norma EN 312 (ECS, 2003). Em vista disso, nenhum dos tratamentos se encaixa nos tipos de painéis citados anteriormente, enquadrando-se apenas no tipo 2, restrito a ambientes internos (incluindo mobiliários), onde não há exigências mínimas em relação ao inchamento em espessura.

\section{Propriedades mecânicas dos painéis MDP}

$\mathrm{Na}$ Figura 4 é possível constatar que houve efeito significativo e negativo entre a porcentagem de resíduos UKP e a ligação interna, isto é, com o aumento da porcentagem de resíduo, ocorreu uma redução dos valores médios da ligação interna.

O decréscimo dos valores de ligação interna com o aumento das porcentagens de resíduos de extração de celulose está relacionado ao processo de colagem das partículas e do UKP, uma vez que os resíduos formaram tufos durante a aplicação do adesivo, dificultando a dispersão do adesivo nas áreas superficiais das partículas utilizadas no miolo. Esse comportamento também foi observado por Guimarães Júnior et al. (2016), com correlação negativa entre a porcentagem de resíduo de sorgo e a ligação interna.

A baixa densidade dos resíduos UKP também pode ter afetado negativamente a ligação interna, pois o aumento no número de partículas acarreta em menor disponibilidade de adesivo por partícula (Protásio et al., 2012; Scatolino et al., 2013). Além disso, a composição química do material também afetou a ligação entre as partículas. Possivelmente, o decréscimo dos valores de ligação interna ocorreu devido aos elevados teores de extrativos e cinzas e baixo de lignina do resíduo UKP. Maiores teores de lignina contribuem com a estabilidade dimensional do painel, devido ao seu caráter hidrofóbico e sua propriedade adesiva (César et al., 2017). Além disso, a quantidade de extrativos, obtido em maior porcentagem para o resíduo de UKP em relação a madeira de Pinus, afeta de forma negativa na cura do adesivo e na formação adequada das ligações mecânicas e específicas (Klímek et al., 2016).

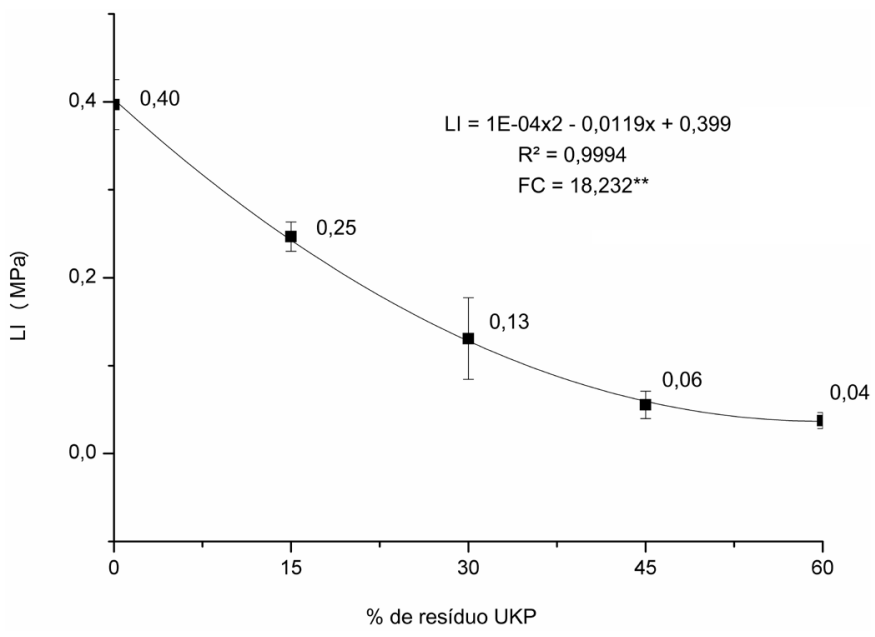

** Análise de regressão significativa ao nível de $1 \%$.

Figura 4. Valores médios de ligação interna dos painéis MDP com resíduo UKP.
Comportamento semelhante ao encontrado neste estudo, onde houve um decréscimo nos valores de ligação interna com o aumento das porcentagens de resíduos agroindustriais, foi observado por outros autores como Scatolino et al. (2013) e Morais et al. (2015).

Apenas o tratamento controle $(0 \%)$ atendeu ao valor mínimo estipulado na EN 312 (ECS, 2003), que é de 0,30 MPa, enquanto os demais tratamentos $(15,30,45$ e $60 \%)$ ficaram abaixo do valor estipulado pela norma citada.

Houve efeito significativo da porcentagem de UKP sobre os módulos de elasticidade - MOE (Figura 5) e de ruptura - MOR (Figura 6) à flexão estática dos painéis. Em ambos os casos, à medida que houve o aumento da porcentagem de resíduo de UKP ocorreu a redução dos valores médios de MOE e MOR, apresentando relação direta com os resultados de ligação interna dos painéis (Figura 4) e os fatores que promoveram sua redução.

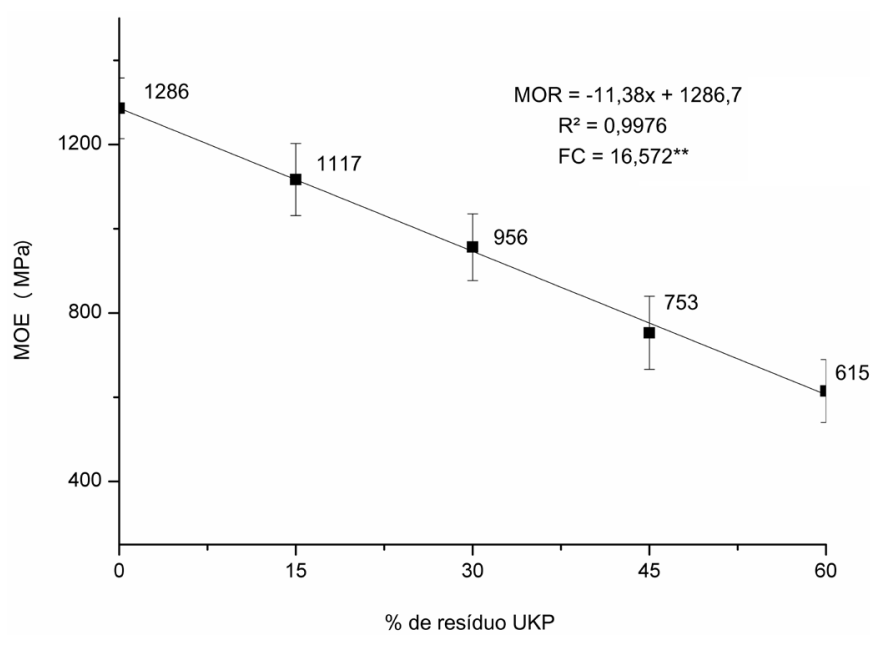

** Análise de regressão significativa ao nível de $1 \%$.

Figura 5. Propriedade módulo de elasticidade à flexão estática para os diferentes níveis de porcentagens de UKP avaliados para a produção de painéis MDP.

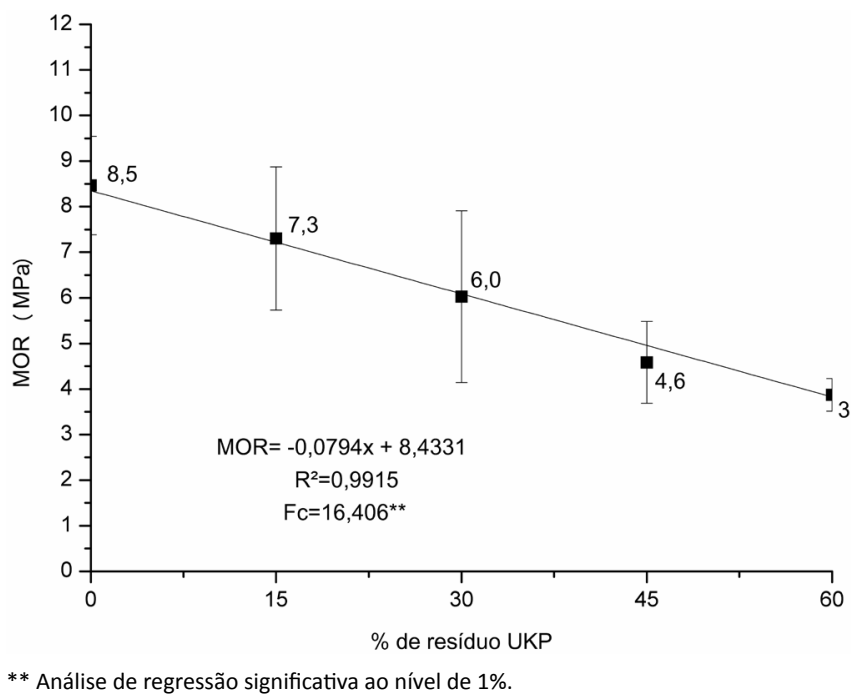

Figura 6. Propriedade módulo de ruptura à flexão estática para os diferentes níveis de porcentagens de UKP avaliados para a produção de painéis MDP. 
A redução dos valores de MOE e MOR pode estar relacionada à baixa densidade do material UKP, o que resulta em um maior número de partículas para se obter uma densidade pré-determinada do painel e, consequentemente, na redução da quantidade de adesivo por partícula (César et al., 2017; Scatolino et al., 2017; Silva et al., 2018).

Mesquita et al. (2015) observaram que houve uma redução do MOE e MOR até $15 \%$ de substituição de eucalipto por sisal nos painéis MDP produzidos, acima dessa porcentagem foi observada certa estabilização do MOE e MOR (substituição de 20 e $25 \%$ por sisal). Os autores explicam que essa redução das propriedades mecânicas pode estar relacionada a fatores de confecção, uma vez que a distribuição das partículas e feixes foi realizada de forma manual, o que dificultou a homogeneização das partículas formando tufos. O mesmo ocorreu na elaboração desta pesquisa, onde a distribuição das partículas também foi realizada de forma manual, acarretando na formação de tufos, prejudicando as propriedades dos painéis.

A NBR 14810-2 (ABNT, 2013), a ANSI A208.1 (ANSI, 1993) e a EN 312 (ECS, 2003) especificam valores mínimos para o módulo de ruptura (MOR) de $16 \mathrm{MPa}, 11,3 \mathrm{MPa}$ e 11,5 $\mathrm{MPa}$, respectivamente. Para o MOE, a norma ANSI A208.1 (ANSI, 1993) especifica o valor mínimo de $1764 \mathrm{MPa}$, enquanto a EN 312 (ECS, 2003) estipula o valor mínimo de $1600 \mathrm{MPa}$. Logo, nenhum dos tratamentos atendeu aos requisitos mínimos para módulo de elasticidade e módulo de ruptura exigidos por essas normas.

\section{Conclusões}

A utilização de partículas de resíduo UKP para a produção de painéis MDP acarretou na diminuição das propriedades mecânicas e no aumento dos valores de absorção de água e inchamento em espessura.

A colagem das partículas foi prejudicada pela composição química do resíduo e pela sua menor densidade, uma vez que houve o aumento da área de contato, gerando uma menor relação adesivo/partícula e afetando diretamente as propriedades físico-mecânicas doa painéis.

Recomenda-se o estudo de quantidade inferior a 15\% para utilização do resíduo UKP como matéria-prima para produção de painéis MDP.

\section{Agradecimentos}

Ao Programa de Pós-graduação em Engenharia de Biomateriais da Universidade Federal de Lavras (UFLA), a Fundação de Amparo à Pesquisa do Estado de Minas Gerais (FAPEMIG), ao Conselho Nacional de Desenvolvimento Científico e Tecnológico (CNPq) e a Coordenação de Aperfeiçoamento de Pessoal de Nível Superior (CAPES).

\section{Literatura Citada}

American National Standard Institute. ANSI A208.1: Mat-formed wood particleboard: Specification, 2009. Gaithersburg: National Particleboard Association, 2009. 9 p.
American Society for Testing and Materials - ASTM. ASTM D 1200: Test method for Viscosity by Ford Viscosity. Philadelphia: ASTM, 1994. 108p.

American Society for Testing and Materials. Annual book of ASTM: D-1037 - standard methods of evaluating properties of wood-base fiber and particles materials. Philadelphia: ASTM, 2016. 32p.

American Society for Testing and Materials. ASTM D1582: adhesives. Washington: ASTM, 1994.

Associação Brasileira de Normas Técnicas - ABNT. NBR 11941: madeira: determinação da densidade básica. Rio de Janeiro: ABNT, 2003. 6 p.

Associação Brasileira de Normas Técnicas - ABNT. NBR 14810: Chapa de madeira aglomerada. Parte 2: Requisitos. Rio de Janeiro: ABNT, 2006. 51p.

Associação Brasileira de Normas Técnicas - ABNT. NBR 14810-1: Painéis de madeira de média densidade. Parte 1: Terminologia. Rio de Janeiro: ABNT, 2013. 5p.

Associação Brasileira de Normas Técnicas - ABNT. NBR 14810-2: Painéis de partículas de média densidade. Parte 2: Requisitos e Métodos de ensaio. Rio de Janeiro: ABNT, 2018. 71p.

Associação Brasileira de Normas Técnicas - ABNT. NBR 7989: Pasta celulósica e madeira - Determinação de lignina insolúvel em ácido. Rio de Janeiro: ABNT, 2010. 6 p.

Associação Brasileira de Normas Técnicas- ABNT. NBR 13999: Papel, cartão, pastas celulósicas e madeira - Determinação do resíduo (cinza) após a incineração a $525^{\circ} \mathrm{C}$. Rio de Janeiro: ABNT, 2003. $4 \mathrm{p}$.

Associação Brasileira de Normas Técnicas. NBR 14853: Madeira - Determinação do material solúvel em etanol-tolueno e em diclorometano e em acetona. Rio de Janeiro, 2010. 3 p.

Bufalino, L.; Albino, V. C. S.; Sá, V. A. de; Corrêa, A. A. R.; Mendes, L. M.; Almeida, N. A. Particleboards made from Australian red cedar: processing variables and evaluation of mixed species. Journal of Tropical Forest Science, v. 24, n. 2, p.162-172, 2012. http://www.jstor.org/stable/23617071. 25 Out. 2018.

Carvalho, A. G.; Andrade, B. G de; Cabral, C. P T.; Vital, B. R. Efeito da adição de resíduos de poda da erva-mate em painéis aglomerados. Revista Árvore, v.39, n.1, p. 209-214, 2015. https:// doi.org/10.1590/0100-67622015000100020.

Cenibra. Departamento de Controle de Qualidade. Caracterização do material fibroso na saída da prensa do decantador primário: relatório interno. Ipatinga: Cenibra, 2008. 8p.

César, A. A. S.; Bufalino, L.; Mendes, L. M.; Mesquita, R. G. de A.; Protásio, T. de P.; Mendes, R. F.; Andrade, L. M. F. Transforming rice husk into a high-added value product: potential for particleboard production. Ciência Florestal, v. 27, n. 1, p. 303 313, 2017. https://doi.org/10.5902/1980509826468.

Consufor. Comportamento da produção mundial de celulose e as oportunidades para o Brasil. https://consufor.com/ comportamento-da-producao-mundial-de-celulose-e-asoportunidades-para-o-brasil. 05 Out. 2018.

Costa, L. P. E. da; Haselein, C. R.; Santini, E. J.; Schneider, P. R.; Calegari, L. Qualidade das chapas de partículas aglomeradas fabricadas com resíduos do processamento mecânico da madeira de Pinus elliottii Engelm. Ciência Florestal, v. 15, n. 3, p. 311-322, 2005. https://doi.org/10.5902/198050981869. 
Deutsches Institut Für Normung - DIN. DIN 52362: testing of wood chipboards bending test, determination of bending strength. Berlin: DIN, 1982. p. 39-40.

European Committee for Standardization - ECS. EN 312: particleboard specifications. Bruxelas: ECS, 2003. 22 p.

Fiorelli, J.; Curtolo, D. D.; Barrero, N. G.; Savastano Júnior, H.; Pallone, E. M de J. A.; Johnson, R. Particulate composite based oncoconut fiber and castor oil polyurethane adhesive: An eco-efficient product. Industrial Crops and Products, v.40, p.69-75, 2012. https://doi.org/10.1016/j.indcrop.2012.02.033.

Gama, R. O.; Dias, F. M; Nascimento, M. F.; Lahr, F. R. Painéis de partículas de rejeito UKP/BKP da indústria de celulose e de resíduos de Pinus elliottii: resistência ao arrancamento de parafusos e resistência à tração perpendicular às faces. Construindo, v. 2, n. 1, p. 35-39, 2010. http://www.fumec.br/ revistas/construindo/article/view/1729. 27 Fev. 2019.

Guimarães Junior, J. B.; Xavier, M. M.; Santos, T. S.; Protásio, T. de P.; Mendes, R. F.; Mendes, L. M. Inclusão de resíduo da cultura de sorgo em painéis aglomerados de eucalipto. Pesquisa Florestal Brasileira, v. 36, n. 88, 2016. https://doi.org/10.4336/2016. pfb.36.88.1036.

Guimarães, B. M. R.; Mendes, L. M.; Tonoli, G. H. D.; Bufalino, L.; Mendes, R. F.; Guimarães Júnior, J. B. Chemical treatment of banana tree pseudostem particles aiming the production of particleboards. Ciência e Agrotecnologia, v. 38, n.1, p. 43-49, 2014. https://doi.org/10.1590/S1413-70542014000100005.

Guimarães, I. L.; Guimarães Júnior, J. B.; Lisboa, F. J. N.; Andrade, C. R.; Siqueira, H. F. Caracterização química e física da madeira de procedências de Eucalyptus. Enciclopédia Biosfera, v. 9, p. 636, 2013. http://www.conhecer.org.br/enciclop/2013b/ CIENCIAS\%20AGRARIAS/caracterizacao\%20quimica\%20e\%20 fisica.pdf. 01 Fev. 2019.

Indústria Brasileira de Árvores - Ibá. Relatório 2018. São Paulo: Ibá, 2018. 5p. https://iba.org/datafiles/publicacoes/relatorios/ digital-sumarioexecutivo-2018.pdf. 26 Fev. 2019.

Iwakiri, S. Painéis de madeira reconstituída. Curitiba: FUPEF, 2005. $254 p$.

Klímek, P.; Meinlschmidt, P.; Wimmer, R.; Plinke, B.; Schirp, A. Using sunflower (Helianthus annuus L.), topinambour (Helianthus tuberosus L.) and cup-plant (Silphium perfoliatum L.) stalks as alternative raw materials for particleboards. Industrial Crops and Products, v. 92, p. 157-164, 2016. https://doi.org/10.1016/j. indcrop.2016.08.004.

Maloney, T. M. Modern particleboard and dry-process fiberboard manufacturing. San Francisco: M. Freeman, 1993. 689 p.

Melo, R.R.; Santini E.J.; Haselein, C.R.; Stangerlin, D.M. Propriedades físico-mecânicas de painéis aglomerados produzidos com diferentes proporções de madeira e casca de arroz. Ciência Florestal, v.19, n.4, p. 449-460, 2009. https://doi. org/10.5902/19805098899.

Mendes, R. F.; Baleeiro, N. S.; Mendes, L. M.; Scatolino, M. V.; Oliveira, S. L.; Protásio, T. de P. Particleboard panels produced with different radial positions of Pinus oocarpa wood. Floresta e Ambiente, v.25, n.1, e00114514, 2018. https://doi. org/10.1590/2179-8087.114514.
Mendes, R. F.; Mendes, L. M.; Guimarães Júnior, J. B.; Mori, F. A.; César, A. A. da Silva. Efeito da incorporação de casca de café nas propriedades físico-mecânicas de painéis aglomerados de Eucalyptus urophylla S. T. Blake. Ciência e Agrotecnologia. v.34, n.3, p.610-617, 2010. https://doi.org/10.1590/S141370542010000300012.

Mendes, R. F.; Mendes, L. M.; Mendonça, L. L.; Guimarães Júnior, J. B.; Mori, F. A. Quality of homogeneous particleboard produced with Eucalyptus urophylla clone wood. Cerne, v.20, n.2, p.329336, 2014. https://doi.org/10.1590/01047760.201420021273.

Mesquita, R. G. A.; Mendes, L. M.; Mendes, R. F.; Tonoli, G. H. D.; Marconcini, J. M. Inclusion of sisal bundles in the production of eucalyptus MDP panels. Scientia Forestalis, Piracicaba, v. 43, n. 105, p. 1-8, 2015. http://www.ipef.br/publicacoes/scientia. 27 Ago. 2018.

Mesquita, R. G. A.; Mendes, L. M.; Sanadi, A. R.; Sena Neto, A. R.; Claro, P. I. C.; Corrêa, A. C.; Marconcini, J. M. Urea Formaldehyde and Cellulose Nanocrystals Adhesive: Studies Applied to Sugarcane Bagasse Particleboards. Journal of Polymers and the Environment, v.26, n.7, p.3040-3050, 2018. https://doi. org/10.1007/s10924-018-1189-4.

Morais, W. W. C.; Haselein, C. R.; Susin, F. Vivian, M. A.; Morais, J. B. F. Mechanical and Physical Properties of Particleboard with Bambusa tuldoides and Pinus taeda. Ciência Florestal, v. 25, n. 4, p. 1015-1026, 2015. https://doi.org/10.5902/1980509820662.

Morais, W. W. C.; Haselein, C. R.; Susin, F.; Vivian, M. A.; Souza, J. T. Uso de Bambusa tuldoides e Eucalyptus grandis para confecção de painéis aglomerados. Ciência Florestal, v. 28, n. 2, p. 746-757, 2018. https://doi.org/10.5902/1980509832088.

Mori, F. A. Caracterização parcial dos taninos das cascas de Eucalyptus para produção de adesivos. Viçosa: Universidade Federal de Viçosa, 2000. 73p. Tese Doutorado.

Moslemi, A.A. Particleboard: materials. Illinois: Southern Illinois University Press, 1974. 244p.

Nakanishi, E. Y.; Cabral, M. R.; Fiorelli, F.; Santos, V.; Christoforo, A. L.; Junior, H. S. Study of the production process of 3-layer sugarcanebamboo-based particleboards. Construction and Building Materials, v. 183 , p. $618-625$, 2018. https://doi.org/10.1016/j. conbuildmat.2018.06.202.

Nemli, G.; Ayan, E.; Ay, N.; Tiryaki, S. Utilization potential of waste wood subjected to insect and fungi degradation for particleboard manufacturing. European Journal of Wood and Wood Products, v. 76, n. 2, p. 759-766, 2018. https://doi.org/10.1007/s00107017-1224-5.

Protásio, T. P.; Guimarães Junior, J. B.; Mendes, R. F.; Mendes, L. M.; Guimarães, B. M. R. Correlações entre as propriedades físicas e mecânicas de painéis aglomerados de diferentes espécies de Eucalyptus. Floresta e Ambiente, v. 19, n. 2, p. 123-132, 2012. https://doi.org/10.4322/floram.2012.014.

Scatolino, M. V. Costa, A.O.; Júnior, J. B. G.; Protásio, T. P.; Mendes, R. F.; Mendes, L. M. Eucalyptus wood and coffee parchment for particleboard production: physical and mechanical properties. Ciência e Agrotecnologia, v. 41, n. 2, p. 139-146, 2017. https:// doi.org/10.1590/1413-70542017412038616. 
Scatolino, M. V.; Silva, D. W.; Mendes, R. F.; Mendes, L. M. Use of cob for production of particleboard. Ciência e Agrotecnologia, v. 37 , n. 4, p. 330-337, 2013. https://doi.org/10.1590/S141370542013000400006.

Silva, D. W.; Farrapo, C.; Ribeiro, D. P.; Scolforo, J. R. S. MDP com partículas de eucalipto e palha de milho. Scientia Forestalis, v.43, n.108, p.853-862, 2015. https://doi.org/10.18671/scifor. v43n108.10.

Silva, D. W.; Scatolino, M. V.; Prado, N. R. T.; Mendes. R. F. Mendes, L. M. Addition of different proportions of Castor husk and Pine wood in particleboards. Waste Biomass Valorization, v. 9, p. 139145, 2018. https://doi.org/10.1007/s12649-016-9742-7.
Soares, S. S.; Guimarães Júnior, J. B.; Mendes, L. M.; Mendes, R. F.; Protásio, T. P.; Lisboa, F. J. N. Valorização do bagaço de cana-de-açúcar na produção de painéis aglomerados de baixa densidade. Ciência da Madeira, v.8, n.2, p. 64-73, 2017. https://doi.org/10.15210/cmad.v8i2.10589.

Sulaiman, N. S.; Hashima, R.; Sulaimana, O.; Nasira, M.; Hazim, M.; Aminib, M. Hizirogluc, S. Partial replacement of urea-formaldehyde with modified oil palm starch based adhesive to fabricate particleboard. International Journal of Adhesion and Adhesives, v. 84, p. 1-8, 2018. https://doi.org/10.1016/j.ijadhadh.2018.02.002.

Xiaodi, J.; Minghui, G. Preparation and properties of a chitosan-lignin wood adhesive. International Journal of Adhesion and Adhesives, v. 82, p. 8-13, 2018. https://doi.org/10.1016/j.ijadhadh.2017.12.005. 